\title{
Endophilin B1 as a Novel Regulator of Nerve Growth Factor/ TrkA Trafficking and Neurite Outgrowth
}

\author{
Jun Wan, ${ }^{1 *}$ Anthony Y. Cheung, ${ }^{1 \star}$ Wing-Yu Fu, ${ }^{1}$ Chengbiao Wu,${ }^{2}$ Mingjie Zhang, ${ }^{1}$ William C. Mobley, ${ }^{2}$ \\ Zelda H. Cheung, ${ }^{1}$ and Nancy Y. Ip ${ }^{1}$ \\ ${ }^{1}$ Department of Biochemistry, Biotechnology Research Institute and Molecular Neuroscience Center, Hong Kong University of Science and Technology, \\ Clear Water Bay, Hong Kong, China, and ${ }^{2}$ Department of Neurology and Neurological Sciences, Pediatrics and Neurosurgery, and Neuroscience Institute, \\ Stanford University, Stanford, California 94305
}

\begin{abstract}
Neurotrophins and their cognate receptors Trks are important regulators of neuronal survival and differentiation. Recent studies reveal that internalization and trafficking of Trks play a critical role in neurotrophin-mediated signaling. At present, little is known of the molecular events that mediate this process. In the current study, we show that endophilin B1 is a novel regulator of nerve growth factor (NGF) trafficking. We found that endophilin B1 interacts with both TrkA and early endosome marker EEA1. Interestingly, knockdown of endophilin B1 results in enlarged EEA1-positive vesicles in NGF-treated PC12 cells. This is accompanied by increased lysosomal targeting of NGF/TrkA and TrkA degradation, and reduced total TrkA levels. In addition, knockdown of endophilin B1 attenuates Erk1/2 activation in the endosomal fraction after NGF treatment. This is accompanied by a marked inhibition of NGF-induced gene transcription and neurite outgrowth in endophilin B1-knocked down cells. Our observations implicate endophilin B1 as a novel regulator of NGF trafficking, thereby affecting TrkA levels and downstream signaling on endosomes to mediate biological functions of NGF.
\end{abstract}

Key words: neurotrophin; Trk receptors; receptor tyrosine kinase; receptor trafficking; neurite outgrowth; endosomes

\section{Introduction}

The neurotrophin family of target-derived growth factors regulates neuronal survival and physiology in both the CNS and PNS. Members of this family include the prototypic member nerve growth factor (NGF), brain-derived neurotrophic factor (BDNF), neurotrophin-3 (NT-3), and NT-4/5. Neurotrophin signaling occurs through the Trk receptor tyrosine kinases (TrkA, $\operatorname{TrkB}$, and TrkC) and the low-affinity neurotrophin receptor p75. Although all neurotrophins bind to p75 with similar affinity, Trk receptors display selectivity toward different neurotrophins. TrkA binds to NGF, whereas TrkB binds preferentially to BDNF and NT-4/5. TrkC, on the other hand, binds to NT-3 (Huang and Reichardt, 2003).

Signaling events mediated by TrkA appear to be determined, at least in part, by trafficking of the receptor. TrkA is internalized after binding with NGF through a P13K (phosphatidylinositol 3 kinase)-dependent mechanism, and is transported retrogradely over long distances from the synapse to the cell body to elicit transcriptional and other somal responses (Neet and Campenot,

Received Feb. 20, 2008; revised July 11, 2008; accepted July 21, 2008.

This work was supported in part by the Research Grants Council of Hong Kong (HKUST 6119/04M, 6431/06M, 661007, and HKUST 3/03C) and the Area of Excellence Scheme of the University Grants Committee (AoE/B-15/01). N.Y.I. and Z.H.C. are Croucher Foundation Senior Research Fellow and Croucher Foundation Fellow, respectively. We thank Winnie Chien, Cong Yu, and Yihang Li for their excellent technical assistance. We are also grateful to Dr. Amy Fu for critical reading of this manuscript and to members of the Ip Laboratory for many helpful discussions.

*J.W. and A.Y.C. contributed equally to this work.

Correspondence should be addressed to Prof. Nancy Y. Ip, Department of Biochemistry, Hong Kong University of Science and Technology, Clear Water Bay, Hong Kong, China. E-mail: boip@ust.hk.

DOI:10.1523/JNEUROSCI.0767-08.2008

Copyright $\odot 2008$ Society for Neuroscience $\quad$ 0270-6474/08/289002-11\$15.00/0
2001; Ginty and Segal, 2002; Howe and Mobley, 2005). Recent studies demonstrated that NGF and activated TrkA are transported in the form of signaling endosomes through microtubulebased transport machinery such as the dynein motors (Delcroix et al., 2003; Ye et al., 2003; Heerssen et al., 2004). These observations indicate that signaling endosomes serve an important role in NGF-mediated neuronal survival.

Endophilin B1 is a new member of the endophilin family that shares significant sequence homology with the well known endocytic protein endophilin A1. Similar to endophilin A1, endophilin B1 exhibits lipid binding and liposome tubulation properties (Farsad et al., 2001). In contrast to endophilin A1, however, endophilin B1 cannot interact with synaptojanin, and is localized mostly to intracellular membranes (Modregger et al., 2003). The functions of endophilin B1 therefore appear to be distinct from that of endophilin A1. Recent studies indicate that endophilin B1, also known as Bif-1 (Bax-interacting factor-1) (Cuddeback et al., 2001), is involved in the regulation of mitochondria morphology and cell death in fibroblasts (Karbowski et al., 2004; Takahashi et al., 2005). In addition, endophilin B1 is recently implicated in the regulation of autophagy in fibroblasts (Takahashi et al., 2007). Nonetheless, the function of endophilin B1 in neurons has remained unknown despite its expression in the brain (Modregger et al., 2003).

In light of the lipid binding property of endophilin B1 and its localization to intracellular membranes, we asked whether endophilin B1 plays a role in endocytic trafficking in neurons. Herein we report that endophilin B1 colocalizes and interacts with endosomal marker early endosomes antigen 1 (EEA1). Importantly, we found that knockdown of endophilin B1 results in 
precocious targeting of internalized NGF/TrkA to the lysosomes, leading to enhanced TrkA degradation and attenuation of NGFTrkA downstream signals on endosomes. Concomitantly, significant inhibition of neurite outgrowth in NGF-treated PC12 cells and superior cervical ganglion (SCG) neurons was observed. Our findings thus provide evidence for a critical role of endophilin B1 in the regulation of NGF trafficking and downstream functions.

\section{Materials and Methods}

DNA constructs and small interfering RNAs, antibodies, and reagents. Fulllength mouse endophilin B1 cDNA was amplified from a mouse brain cDNA library. The cDNA fragment was inserted into mammalian expression vectors pEGFP-N2 (Clontech) and pcDNA3 (Invitrogen). DNA sequencing was performed using an ABI310 genetic analyzer (Applied Biosystems). Small interfering RNAs (siRNAs) oligos were purchased from Invitrogen. Sequences of the siRNAs are as follows: endophilin B1 siRNA, 5'-UAAGUAGGUAAUUGGCACCUUGCCC; endophilin B1 scrambled siRNA, 5'-UAAGUGGGAUUAAUCGGUUCCACCC. The vector-based endophilin B1 small hairpin RNAs (shRNAs) were constructed by inserting the following double-stranded oligonucleotides into the pSUPER vector between BglII and XhoI sites: mouse/rat endophilin B1 (N terminus, base pairs 548-566) forward, 5'-GATCTCCAAGCTGCAGAAACTAAAAGTTCAAGAGACTTTTAGTTTCTGCAGCTTTTTTTGGAAC (both sense and antisense endophilin B1 sequences are underlined). shRNA-resistant endophilin B1 was generated by mutating AAGCTGCAGAAACTAAAAGT (base pairs 548-567 of EndoB1) to AAGCAGCTGAGACAAAGTCC without changing amino acid sequence. Antibodies used in the current study include mouse monoclonal antibodies to EEA1 (BD Biosciences), TrkA (MCTrks) (Santa Cruz Biotechnology), $\alpha$-tubulin (Santa Cruz Biotechnology), $\beta$-tubulin III (Sigma-Aldrich), lysosome-associated membrane protein 2 (LAMP2) (ABL-93) (Department of Pharmacology and Molecular Sciences, Johns Hopkins University School of Medicine, Baltimore, MD); and rabbit polyclonal antibodies Rab7 (H-50) (Santa Cruz Biotechnology), Rab5 (Santa Cruz Biotechnology), Rab4 (BD Biosciences), Rab11 (Santa Cruz Biotechnology), Pan-Endophilin (Zymed), Trk (C-14) (Santa Cruz Biotechnology), phospho-Akt (Thr308), Akt (Cell Signaling), phospho-p42/p44, p42/p44 (Cell Signaling), Rap1 (Santa Cruz Biotechnology), and CD71 (transferrin receptor; Santa Cruz Biotechnology). Endophilin B1 antibody was raised in rabbit using a peptide $\left(\mathrm{NH}_{2}\right.$-CGNQKGKVPITYLELL-OH; Bio-Synthesis) encoding endophilin B1 C terminus (amino acids 350-365). The resulting serum was purified using the same peptide coupled to a Sulfolink column (Pierce Biotechnology). Sulfo-NHS-LC-biotin and sulfo-NHS-SS-biotin were purchased from Pierce.

Cell cultures and transfection. PC12 cells were maintained in DMEM with $6 \%$ heat-inactivated fetal bovine serum, $6 \%$ heat-inactivated horse serum, $100 \mathrm{U} / \mathrm{ml}$ penicillin, and $100 \mu \mathrm{g} / \mathrm{ml}$ streptomycin in a $37^{\circ} \mathrm{C}$ incubator with $7.5 \% \mathrm{CO}_{2}$. Medium was changed every $2 \mathrm{~d}$. For NGF-induced differentiation, cells were plated $1 \mathrm{~d}$ before treatment with the NGF, and serum concentration was reduced to $1 \%$. NGF (Alomone Labs) was added at the concentration of $50 \mathrm{ng} / \mathrm{ml}$. Medium was changed every $2 \mathrm{~d}$ and supplemented with additional NGF. Primary hippocampal neuron cultures were prepared from embryonic day 18 rat embryos. Hippocampal neurons were plated on poly-D-lysine-coated culture plates in Neurobasal medium containing B27 supplement, penicillin $(50 \mathrm{U} / \mathrm{ml})$, and streptomycin $(100 \mu \mathrm{g} / \mathrm{ml})$. SCGs were dissociated from postnatal day 1 rats and cultured as described previously ( $\mathrm{Ng}$ et al., 2003). Cultures were incubated in a $37^{\circ} \mathrm{C}$ incubator with $5 \% \mathrm{CO}_{2}$. $\mathrm{PC} 12$ cells were transfected with various plasmids or siRNAs using Lipofectamine 2000 (Invitrogen) according to the manufacturer's instruction. Dissociated SCG neurons were transfected with a primary neuron nucleofection kit (Amaxa Biosystems) according to the supplier's instructions.

Preparation of cell extracts and Western blot analysis. PC12 cells were harvested and lysed in radioimmunoprecipitation assay (RIPA) lysis buffer [ $150 \mathrm{~mm} \mathrm{NaCl}, 1 \%$ (v/v) Nonidet P-40, 0.1\% (w/v) SDS, $2 \mu \mathrm{g} / \mathrm{ml}$ aprotinin, $0.5 \%$ deoxycholic acid, $1 \mathrm{~mm}$ phenylmethylsulfonyl fluoride, 5 $\mathrm{mm}$ benzamidine, $1 \mathrm{~mm}$ sodium orthovanadate (NaOV), and $10 \mu \mathrm{g} / \mathrm{ml}$ soybean trypsin inhibitor] in $50 \mathrm{~mm}$ phosphate buffer, $\mathrm{pH}$ 8.0. Brain tissues were homogenized in lysis buffer B (50 mM Tris, $\mathrm{pH} 8,150 \mathrm{~mm}$ $\mathrm{NaCl}, 2$ mm EGTA, 1 mm dithiothreitol, $1 \%$ Nonidet P-40, $0.25 \%$ sodium deoxycholate, $50 \mathrm{~mm} \mathrm{NaF}$ ) with various protease inhibitors. The proteins separated by SDS-PAGE were then transferred onto a nitrocellulose membrane (GE Osmonics) and probed with various antibodies. Proteins were visualized using the Supersignal West Pico Chemiluminescent Substrate kit (Pierce).

Subcellular fractions preparation. The 10 subcellular fractions of adult rat brain were prepared as previously described (Huttner et al., 1983; Niethammer et al., 2000; Liu et al., 2006). Briefly, rat brain was homogenized in $0.32 \mathrm{M}$ sucrose buffer (fraction $\mathrm{H}$ ) and centrifuged at $800 \times g$ to pellet nuclei and large cellular debris (P1). The supernatant (S1) was subjected to $10,000 \times g$ centrifugation to yield the crude synaptosomal fraction (P2). The supernatant was centrifuged at $165,000 \times g$ for $2 \mathrm{~h}$, resulting in cytosol (S3) and light membrane (P3) fractions. The synaptosomal fraction $\mathrm{P} 2$ was lysed hypoosmotically and spun at 25,000 $\times g$ for $20 \mathrm{~min}$ to obtain the synaptosomal membrane fraction LP1. The supernatant, LS1, was centrifuged at $165,000 \times g$ for $2 \mathrm{~h}$, resulting in a synaptic vesicle enriched fraction (LP2) polyacryl and a supernatant (LS2).

Preparation of the endosomal fraction. PC12 cells fractionation was performed by using the method described by Wu et al. (2001). Briefly, NGF-treated PC12 cells were washed once by cold PBS and harvested by centrifugation $(800 \times g$ for $10 \mathrm{~min})$. Cells were homogenized in $0.5 \mathrm{ml}$ of cold homogenization buffer (HB) (in mм: 250 sucrose, 20 Tricine$\mathrm{NaOH}, \mathrm{pH} 7.8,1 \mathrm{EDTA}$, and $2 \mathrm{MgCl}_{2}$ ). After centrifugation at $800 \times g$ for $10 \mathrm{~min}, 0.5 \mathrm{ml}$ of $50 \%$ OptiPrep in $\mathrm{HB}$ was added to the supernatant to obtain a $1 \mathrm{ml}$ mixture in $25 \%$ OptiPrep. The $25 \%$ Optiprep was placed at the bottom of a centrifuge tube and overlaid with $1 \mathrm{ml}$ each of 20, 15, 10 , and $5 \%$ OptiPrep in cold HB. After centrifugation in a Hitachi preparative ultracentrifuge (Himac CP80MX) with a Sorvall TH 641 rotor at $100,000 \times g$ at $4^{\circ} \mathrm{C}$ for $18 \mathrm{~h}$, membrane fractions were collected from each of the four interphases of the OptiPrep gradients.

Fluorescence microscopy studies. PC12 cells were fixed with 4\% paraformaldehyde for $30 \mathrm{~min}$ at room temperature. After blocking with $4 \%$ goat serum and $1 \%$ BSA in PBS containing $0.4 \%$ Triton X-100 for 30 min at room temperature, cells were incubated with various primary antibodies $(1: 500-1: 2000)$ as indicated at $4^{\circ} \mathrm{C}$ overnight. Cells were washed with PBS three times. Alexa Fluor 488-, Alexa Fluor 568-, or Alexa Fluor 647-conjugated anti-mouse or anti-rabbit IgG (Invitrogen; 1:1000) were added and incubated for $1 \mathrm{~h}$ at room temperature. After extensive washing with PBS, cells were mounted with anti-Fade reagent (Invitrogen) and analyzed under Olympus confocal microscope (Fluoview BX61; Olympus). Quantification of immunoreactivity colocalization was performed according to the protocol by Volpicelli et al. (2002).

Biotinylation assays. Biotinylation assays were performed as described previously (Kuruvilla et al., 2004; Rong et al., 2006). For biotinylation of membrane TrkA, PC12 cells were transfected with scrambled or endophilin B1 siRNAs. Forty-eight hours after transfection, cells were serum starved overnight followed by incubation with NGF $(50 \mathrm{ng} / \mathrm{ml})$ in DMEM containing $1 \%$ horse serum at $37^{\circ} \mathrm{C}$ for different time periods indicated. Cells were then washed three times with ice-cold PBS containing $1 \mathrm{~mm} \mathrm{CaCl}_{2}$ and $0.5 \mathrm{~mm} \mathrm{MgCl}_{2}$ (PBS-Ca-Mg) and incubated with sulfo-NHS-LC-biotin $\left(0.5 \mathrm{mg} / \mathrm{ml}\right.$ in PBS-Ca-Mg) for $30 \mathrm{~min}$ at $4^{\circ} \mathrm{C}$. PBS-Ca-Mg containing $100 \mathrm{~mm}$ glycine as the quenching buffer was added to biotinylated cells for $15 \mathrm{~min}$ at $4^{\circ} \mathrm{C}$ followed by PBS-Ca-Mg wash twice before cell lysis. PC12 cells were lysed in RIPA lysis buffer as described above. Streptavidin beads (Pierce) were incubated with $500 \mu \mathrm{g}$ cell extracts for $2 \mathrm{~h}$ at $4^{\circ} \mathrm{C}$. After extensive washing with the RIPA buffer, bound proteins were eluted by boiling in SDS-PAGE sample buffer and subjected to SDS-PAGE and immunoblotting. The supernatant obtained after immunoprecipitation was also probed with $\alpha$-tubulin antibody as the loading control.

Intracellular trafficking of TrkA receptor labeled by Quantum dot. Qdot605 streptavidin conjugate was purchased from Invitrogen. Biotinylated NGF was generated according to the method described by Bronfman et al. (2003). Briefly, NGF and amino-biotin were dissolved together in MES-NaOH buffer, pH 5.0. After EDC [1-ethyl-3-(3-dimethyl amin- 
opropyl)carbodiimide] was added, the solution was shaken at room temperature for $2 \mathrm{~h}$. Biotinylated NGF was then purified by reverse-phase HPLC over a C4 column (Vydac) and quantified by spectroscopy. Two days after transfection of PC12 cells, the cells were serum starved overnight. Qdot-605 and biotinylated-NGF were incubated in DMEM for 40 min on ice and diluted in ice-cold DMEM to attain a final Quantum dot (Qdot) concentration of $1 \mathrm{~nm}$. Serum-starved PC12 cells were cooled down at $4^{\circ} \mathrm{C}$ for $30 \mathrm{~min}$, and then washed with ice-cold PBS twice. DMEM containing Qdot-NGF was added to cells and incubated at $4^{\circ} \mathrm{C}$ for $30 \mathrm{~min}$. After incubation, the cells were washed three times with ice-cold PBS, followed by incubation with fresh DMEM at $37^{\circ} \mathrm{C}$ for different time periods. The cells were then fixed, immunostained with indicated antibodies, and analyzed under Olympus confocal microscope. The number of intracellular Qdot fluorescent vesicles after different periods of Qdot-NGF treatment was counted and the percentage of Qdot vesicles that colocalized with different proteins was calculated. At least 50 cells per dish were counted. Each experiment was repeated at least three times.

Intracellular trafficking of transferrin receptor. Biotin-labeled transferrin and streptavidin Alexa 555 were purchased from Sigma-Aldrich and Invitrogen, respectively. Two days after transfection of PC12 cells, the cells were serum starved overnight. Biotin-labeled transferrin and streptavidin Alexa 555 were incubated in PBS for $30 \mathrm{~min}$ on ice and diluted in ice-cold DMEM to attain a final transferrin concentration of $100 \mu \mathrm{g} / \mathrm{ml}$. The following procedures were the same as those described in Qdot-NGF-TrkA trafficking experiment.

Quantitative RT-PCR. Total RNA was isolated from NGF-treated and untreated PC12 cells using RNeasy mini kit (QIAGEN) according to the manufacturer's instruction. First-strand cDNA was synthesized with ImProm-II reverse transcription system (Promega). Quantitative PCR was performed with Bio-Rad MX3000P real-time PCR system.

Quantification of neurite outgrowth. In the morphological study of PC12 cells, length of the longest neurite and total neurite length were measured to quantify neurite outgrowth using MetaMorph version 5.0r1 software (Molecular Devices). For each measurement, at least 100 cells per dish were counted from randomly selected fields. Each experiment was repeated at least three times. The total number of tip ends was counted to represent the number of neurites from individual cells ( $\mathrm{Ng}$ et al., 2003).

Statistical analysis. All data were expressed as mean \pm SD. Statistical significance was determined by one-way ANOVA followed by Tukey's post hoc test with $95 \%$ confidence. A value of $p<0.05$ was considered to be statistically significant.

\section{Results \\ Endophilin B1 colocalized and interacted with EEA1 in neurons}

To explore the potential functions of endophilin B1 in neurons, we began by investigating the expression of endophilin B1 in the developing brain and SCGs. Endophilin B1 expression increased during development and maximal level was detected in the adult brain and SCG (Fig. 1A). To examine the subcellular distribution of endophilin B1, adult brain was subjected to fractionation. We found that endophilin B1 was enriched in a light membrane fraction, which includes plasma membrane and intracellular membranes, such as endosomes and lysosomes. In addition, endophilin B1 was also found in a fraction enriched in synaptic vesicles (Fig. $1 B$ ). In contrast, endophilin A appeared to be expressed nearly ubiquitously (Fig. $1 B$ ).

The localization of endophilin B1 to light membrane fraction and its ability to bind lipids strongly suggest that it may be implicated in the endocytic pathways. The endocytic pathway commences at the plasma membrane from which cargo is internalized and transported to early endosomes. Subsequent events include the maturation of these endosomes into late endosomes (Miaczynska and Zerial, 2002). Proteins transported to late endosomes are often targeted to lysosomes where they are degraded (Gáborik and Hunyady, 2004). To examine the possible involvement of endophilin B1 in the endocytic pathway, we asked whether endophilin B1 colocalized with specific endocytic compartments using immunocytochemistry. Interestingly, although endogenous endophilin B1 appeared to colocalize partially with both early endosome marker EEA1 and LAMP2 in the cell body of cultured hippocampal neurons, colocalization with EEA1 occurred in clear puncta, whereas only diffuse colocalization was observed between endophilin B1 and LAMP2 (Fig. 1C). Furthermore, endophilin B1 colocalized with EEA1, but not LAMP2, in the neurites of the hippocampal neurons (Fig. 1C). Indeed, quantification of the colocalization of endophilin B1 staining with EEA1 and LAMP2 immunoreactivities confirmed that the percentage of colocalization between EEA1 and endophilin B1 staining was markedly higher than that between LAMP2 and endophilin B1 (Fig. 1C). Quantification of the colocalization of endophilin B1 immunoreactivity with EEA1 or LAMP2 staining in PC12 cells yielded comparable results (data not shown). These observations collectively indicate that endophilin B1 was expressed in neurons and colocalized with EEA1 in puncta in both cell body and processes, and also to a lesser extent with LAMP2 in the cell body.

To substantiate the involvement of endophilin B1 in the endocytic pathway, we examined the association of endophilin B1 with various markers of the endocytic pathways in PC12 cells before and after NGF treatment. Interestingly, we found that endophilin B1 interacted only with EEA1, but not LAMP2, Rab4 (marker for recycling endosomes), or Rab7 (marker for late endosomes). Furthermore, interaction between endophilin B1 and EEA1 was enhanced in the presence of NGF (Fig. 1D). Our observations indicate that endophilin B1 was specifically associated with EEA1-positive vesicles, thus suggesting a potential role of endophilin B1 in the endocytic pathways in neurons, possibly at the level of early endosomes.

\section{Knockdown of endophilin B1 reduced targeting of NGF and TrkA to EEA1-positive vesicles and resulted in enlargement of EEA1-positive vesicles after NGF treatment}

To test for the involvement of endophilin B1 in the endocytic pathway, we examined the trafficking of two surface receptors, TrkA and transferrin receptors in PC12 cells. Trk activation is followed by internalization and trafficking through the endocytic pathway (Delcroix et al., 2003; Ye et al., 2003), which has emerged as an important regulator of the downstream signaling cascades and the functional responses induced by neurotrophin stimulation (Ye et al., 2003; Heerssen et al., 2004). Transferrin receptor, however, is mostly recycled subsequent to internalization (Miaczynska and Zerial, 2002). Although it has been previously demonstrated that endophilin B1 has no effect on transferrin endocytosis in fibroblasts (Modregger et al., 2003), whether endophilin B1 affects transferrin receptors trafficking through the endocytic pathway remains unknown. Given the interaction of endophilin B1 with EEA1 and its localization to EEA1- and LAMP2-positive vesicles, it is of interest to examine whether endophilin B1 affects the trafficking of Trk receptor and transferrin receptor.

To determine whether endophilin B1 plays a role in TrkA trafficking, we first examine whether endophilin B1 and TrkA colocalize in PC12 cells. Both before and after NGF treatment, endophilin B1 colocalized partially with TrkA (Fig. 2 A), although colocalization was enhanced after NGF treatment. In agreement with this observation, endophilin B1 interacted with TrkA in the presence or absence of NGF, with NGF treatment further aug- 
A.

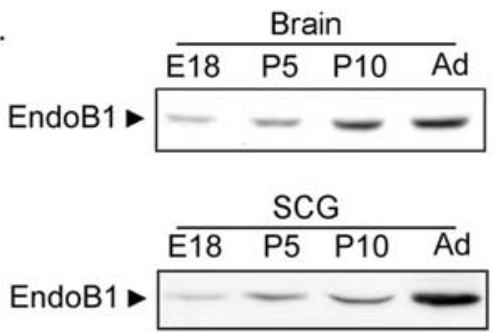

C.
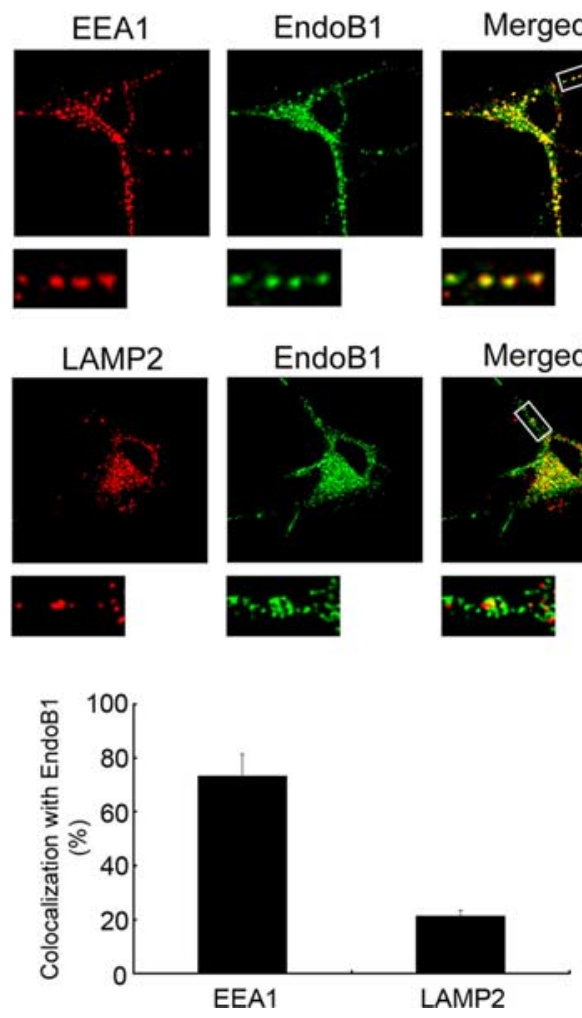

B.

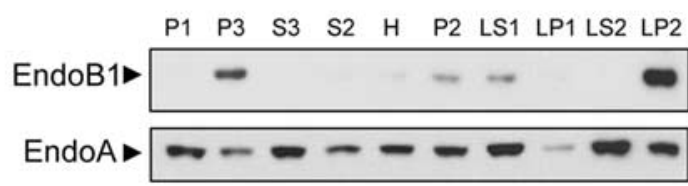

D.

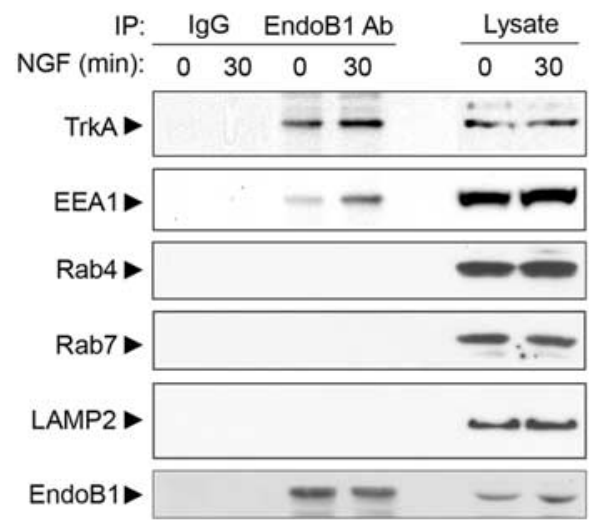

Figure 1. Endophilin B1 was localized to EEA1-positive early endosomes. $\boldsymbol{A}$, Expression of endophilin B1 was developmentally regulated in the brain (top) and SCGs (bottom). Expression of endophilin B1 increased throughout development, with maximal expression detected in the adult stage (Ad). $\boldsymbol{B}$, Endophilin B1 was localized to light membrane and synaptic vesicle-enriched fractions. Adult brains were subjected to differential centrifugations to obtain various fractions. Immunoblotting against endophilin B1 revealed that endophilin B1 was localized to a light membrane fraction (P3), which includes plasma membrane and intracellular organelles such as endosomes and lysosomes. In addition, endophilin B1 was enriched in a synaptic vesicle-enriched fraction (LP2), indicating that endophilin B1 may also be present in synaptic vesicles. Endophilin A (EndoA) was included as controls. C, Endogenous endophilin B1 colocalized partially with EEA1 and LAMP2 in the cell body of hippocampal neurons, but punctate colocalization was only observed between endophilin B1 and EEA1 in the neurites. Quantitation of the colocalization between endophilin B1 staining and EEA1 or LAMP2 immunoreactivities is depicted in the histogram. Error bars indicate SD. Scale bar, $20 \mu \mathrm{m}$. D, Endophilin B1 interacted with EEA1, but not Rab4, Rab7, and LAMP2 in PC12 cells. Association between EEA1 and endophilin B1 was enhanced after 30 min of NGF treatment. Endophilin B1 also interacted with TrkA both before and after NGF treatment, although NGF treatment further enhanced the association.

menting their association (Fig. 1D). In contrast, we found that TrkA colocalized partially with early endosome markers EEA1 only after NGF treatment (Fig. 2A). Quantitation of the percentage of colocalization between TrkA and EEA1 staining confirmed that colocalization between TrkA and EEA1 increased markedly after NGF treatment (Fig. $2 A$ ). These observations indicate that NGF treatment may trigger internalization of TrkA into EEA1positive early endosomes, consistent with previous observations (Wu et al., 2001). Our findings suggest that TrkA, endophilin B1, and EEA1 may all be localized to early endosomes after NGF treatment.

To investigate the potential role of endophilin $\mathrm{B} 1$ in NGF/ TrkA trafficking, endophilin B1 expression was knocked down using the siRNA approach. As shown in Figure $2 B$, transfection of endophilin B1 siRNA oligonucleotides nearly abolished endophi- lin B1 expression in PC12 cells compared with those transfected with scrambled siRNA oligonucleotides. PC12 cells transfected with scrambled or endophilin B1 siRNA were treated with Qdotconjugated NGF for 15,30 , or $60 \mathrm{~min}$. The use of Qdot-NGF allowed visualization of internalized NGF and has been shown to colocalize with TrkA in the cell bodies and axon of neurons (Cui et al., 2007). We also found that internalized Qdot-NGF colocalized extensively with TrkA in PC12 cells during the entire period of NGF treatment (95-100\% colocalization for all treatment groups) (data not shown), indicating that most of the Qdot-NGF was present in vesicles containing TrkA (Fig. 2C). Subsequent to NGF treatment, an increasing amount of Qdot-NGF and TrkA colocalized with EEA1-positive vesicles in control cells, with colocalization still evident after 60 min of NGF treatment. This is supported by quantification of the percentage of Qdot/TrkA co- 


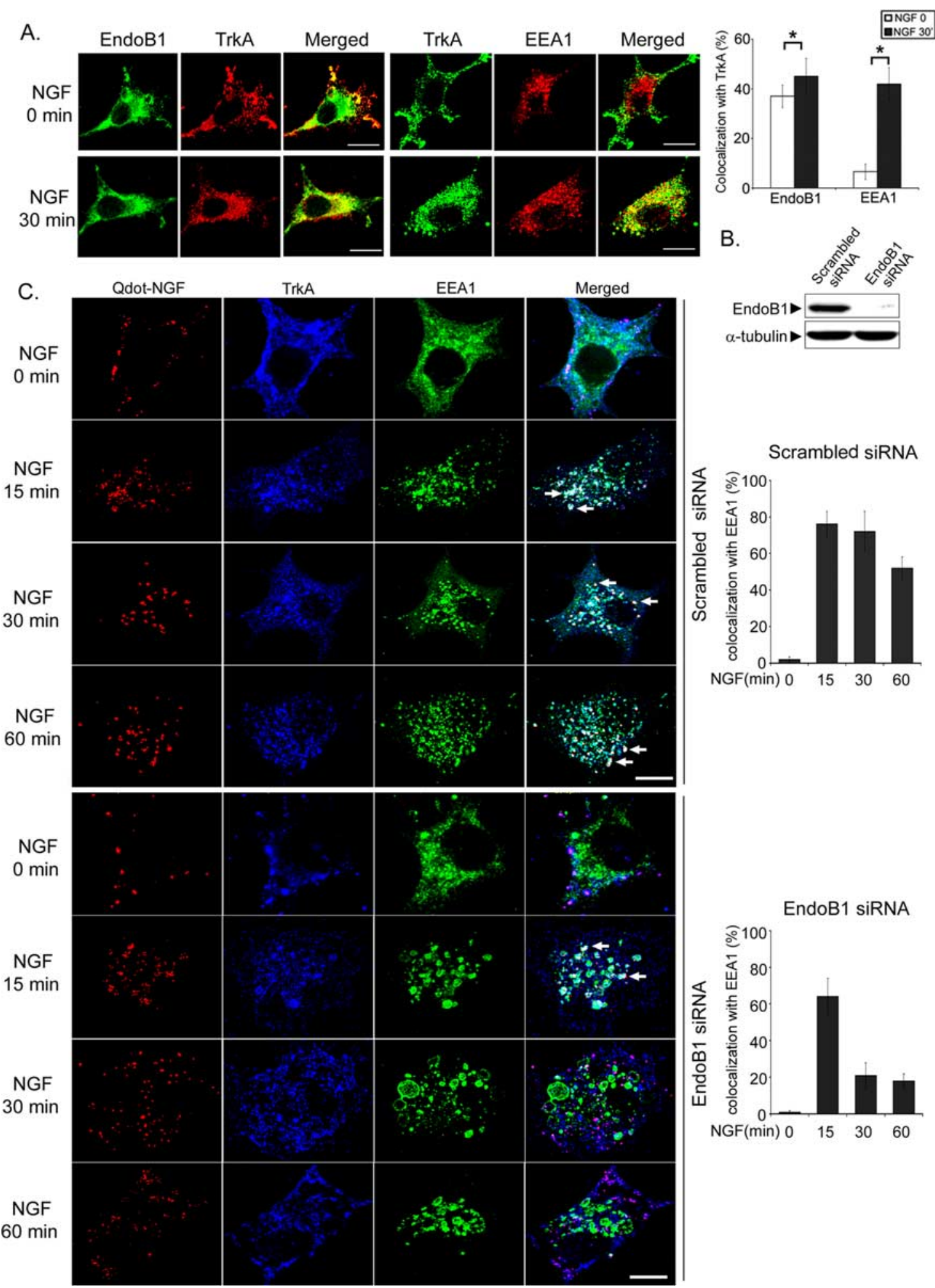

Figure 2. Knockdown of endophilin B1 reduced trafficking of NGF to EEA1-positive vesicles and resulted in the formation of enlarged EEA1-positive vesicles after NGF treatment. $A$, Endophilin B1 colocalized with TrkA and EEA1 after NGF treatment. PC12 cells were treated with NGF $(50 \mathrm{ng} / \mathrm{ml})$, and the localization of endophilin B1, TrkA, and EEA1 was examined using immunocytochemistry. We found that endophilin B1 (endoB1) colocalized with TrkA before and after NGF treatment, although TrkA colocalized with EEA1 only after NGF treatment. Quantitation of the colocalization between TrkA staining and endophilin B1 or EEA1 immunoreactivities before and after NGF treatment is depicted in the histogram. Error bars indicate SD. ${ }^{*} p<0.05$. $\boldsymbol{B}$, Knockdown of endophilin B1 expression using siRNA nearly abolished endophilin B1 expression in PC12 cells. PC12 cells were transfected with scrambled or endophilin B1 siRNA. Western blot analysis revealed that transfection of endophilin B1 siRNA was effective in reducing endophilin B1 expression in PC12 cells. C, Knockdown of endophilin B1 expression reduced targeting of NGF and TrkA to EEA1-positive vesicles and resulted in the formation of enlarged EEA1-positive endosomes after NGF treatment. PC12 cells were transfected with scrambled or endophilin B1 siRNA, and then pretreated with Qdot-NGF for 15 min at $4^{\circ} \mathrm{C}$ to allow binding to surface TrkA, followed by incubation at $37^{\circ} \mathrm{C}$ for different periods to observe internalization of Qdot-NGF/TrkA. Qdot-NGF colocalized extensively with TrkA (purple entities in the merge panel) before incubation at $37^{\circ} \mathrm{C}$, indicating that Qdot-NGF was associated with TrkA. After the initiation of Trk internalization, colocalization of Qdot-NGF, TrkA, and EEA1 was observed (white spots in the merge panel; indicated by arrows). Knockdown of endophilin B1 markedly reduced localization of Qdot-NGF/TrkA to EEA1-postivie vesicles at 30 and 60 min. Furthermore, knockdown of endophilin B1 resulted in the formation of enlarged EEA1-positive vesicles that colocalized with TrkA and Qdot-NGF after 15 min of NGF treatment. Scale bars, $10 \mu \mathrm{m}$. 
localization with EEA1 (Fig. 2C). Interestingly, knockdown of endophilin B1 expression reduced the colocalization of QdotNGF and TrkA with EEA1-positive vesicles at 30 and $60 \mathrm{~min}$, compared with the scrambled-siRNA transfected cells (Fig. 2C). Indeed, the percentage of colocalization between Qdot/TrkA and EEA1 was reduced from $50-70 \%$ at $30-60$ min of NGF treatment in control cells to $\sim 20 \%$ in endophilin B1 siRNA-transfected cells (Fig. 2C). In contrast, examination of Qdot-NGF trafficking in PC12-nnr5 cells, which express only p75, revealed that trafficking of Qdot-NGF was not affected by knockdown of endophilin B1 (supplemental Fig. 1, available at www.jneurosci.org as supplemental material). Furthermore, knockdown of p75 expression through transfection of a p75 siRNA had no effect on the trafficking of Qdot-NGF in PC12 cells (supplemental Fig. 1, available at www.jneurosci.org as supplemental material). These observations collectively indicate that endophilin B1 knockdown specifically affected trafficking of Qdot-NGF bound to TrkA or TrkAcontaining complex, but not p75.

Remarkably, although the size of EEA1-positive vesicles was comparable between the scrambled siRNA and endophilin B1 siRNA-transfected groups before NGF treatment, NGF treatment resulted in the formation of enlarged EEA1-positive vesicles in endophilin B1-knocked down cells (Fig. 2C). Enlargement was particularly evident after $15-60$ min of NGF treatment. Our observations thus reveal a role of endophilin B1 in the trafficking of Qdot-NGF and TrkA through EEA1-positive vesicles and raise the possibility that endophilin B1 may control the size of NGF/ TrkA-containing early endosomes.

To examine whether endophilin B1 similarly affects trafficking of transferrin and transferrin receptor, we first examined whether endophilin B1 interacts with transferrin receptor. In contrast to TrkA, no association was detected between endophilin $\mathrm{B} 1$ and transferrin receptor, whether in the presence or absence of transferrin stimulation (supplemental Fig. $2 \mathrm{~A}$, available at www.jneurosci.org as supplemental material). Furthermore, knockdown of endogenous endophilin B1 level using the siRNA approach had no effect on transferrin receptor level (supplemental Fig. $2 B$, available at www.jneurosci.org as supplemental material) or trafficking of Alexa 555-conjugated transferrin through the endocytic pathway in PC12 cells (supplemental Fig. 2C, available at www.jneurosci.org as supplemental material). Our observations therefore demonstrated that, although endophilin B1 is involved in the trafficking of TrkA, it plays a negligible role in the trafficking of transferrin/transferrin receptor subsequent to internalization.

\section{Knockdown of endophilin B1 favored trafficking of NGF/ TrkA to late endosomes and lysosomes}

To characterize the role of endophilin B1 in NGF/TrkA trafficking in the endocytic pathway, PC12 cells transfected with scrambled or endophilin B1 siRNA were treated with QdotNGF, fixed, and immunostained against EEA1, Rab7, and LAMP2. We found that, in scrambled siRNA-transfected cells, Qdot-NGF was localized almost exclusively to EEA1-positive early endosomes after $15 \mathrm{~min}$ of Qdot-NGF stimulation. Colocalization of Qdot-NGF with late endosome marker Rab7 increased gradually, peaking at $45 \mathrm{~min}$. By $60 \mathrm{~min}$, a large portion of Qdot-NGF colocalized with LAMP2-positive vesicles: this was accompanied by a marked reduction in colocalization with EEA-1-positive vesicles (Fig. $3 A, B$ ). Remarkably, transfection of endophilin B1 siRNA resulted in precocious trafficking of Qdot-NGF to late endosomes and lysosomes. The percentage of Qdot-NGF that colocalized with EEA1 was markedly reduced from 30 to $60 \mathrm{~min}$, as demonstrated in Figure $2 C$, and there was a concomitant increase in Qdot-NGF targeting to late endosome and lysosome (Fig. $3 A, B$ ). These findings suggest that knockdown of endophilin B1 leads to premature trafficking of Qdot-NGF, possibly in a complex with TrkA, to late endosome and lysosomes.

\section{Knockdown of endophilin B1 triggered accelerated degradation of TrkA}

Because knockdown of endophilin B1 resulted in precocious lysosomal targeting of Qdot-NGF, we considered the possibility that knockdown of endophilin B1 would accelerate degradation of TrkA, given the colocalization of TrkA with Qdot-NGF (Fig. $2 C)$. To further pursue this idea, we examined TrkA levels after transfection of endophilin B1 siRNA in PC12 cells. We found that knockdown of endophilin B1 markedly reduced total TrkA levels before and after NGF treatment (Fig. $4 A$ ). Furthermore, by normalizing against the respective TrkA level before NGF treatment in control and endophilin B1 knockdown cells, we found that NGF-induced downregulation of total TrkA was accelerated in endophilin B1 knockdown cells (Fig. 4A). In addition, reduction of surface TrkA was similarly accelerated in endophilin B1 knockdown cells (Fig. 4A). Our observations thus reveal that knockdown of endophilin B1 reduced both basal total and surface TrkA levels, and accelerated NGF-induced downregulation of TrkA in PC12 cells.

\section{Knockdown of endophilin B1 inhibited TrkA downstream signaling at endosomes and NGF-induced gene transcription} Because knockdown of endophilin B1 markedly reduced TrkA level and accelerated NGF-induced TrkA downregulation, we were interested to determine whether endophilin B1 affects TrkA signaling. To answer this question, we first examined whether knockdown of endophilin B1 affected NGF-triggered TrkA signaling in PC12 cells using total lysates. Intriguingly, despite the reduction in total TrkA level in endophilin B1-knocked down cells, downstream signaling initiated in response to NGF treatment such as Erk1/2 and Akt activation was not significantly affected (Fig. $4 B$ ). We thus proceeded to examine whether reduction of endophilin B1 levels affected TrkA signaling from endosomes. Using a fractionation protocol that allows isolation of endosomes-enriched membrane fractions, endosomal fractions were isolated from PC12 cells transfected with scrambled or endophilin B1 siRNA after treatment with NGF for $30 \mathrm{~min}$. The presence of Rap-1, a marker for signaling endosomes, in the endosomal fraction (Fig. 4C, fraction 4) verified the enrichment of endosomes in this fraction. Similar to what was observed using total lysates (Fig. 4A), we found that knockdown of endophilin B1 reduced total TrkA levels in endosomal fraction. In addition, pTrkA level was also significantly lower compared with scrambled siRNA-transfected cells. More importantly, phospho- and total Erk1/2 levels in the endosomal fraction were significantly reduced in endophilin B1-knocked down cells after NGF treatment, although phospho- and total-Akt levels remained mostly unchanged (Fig. 4C). Our observations revealed that knockdown of endophilin B1 attenuated TrkA level and initiation of Erk signaling in endosomes, but not in total lysates, after NGF treatment, and may affect recruitment of signaling molecules to endosomes.

In light of the reduced TrkA level and attenuated Erk activation in endosomes after knockdown of endophilin B1, we tested whether knockdown of endophilin B1 affects NGF-induced gene transcription using quantitative real-time PCR. In accordance 
A.
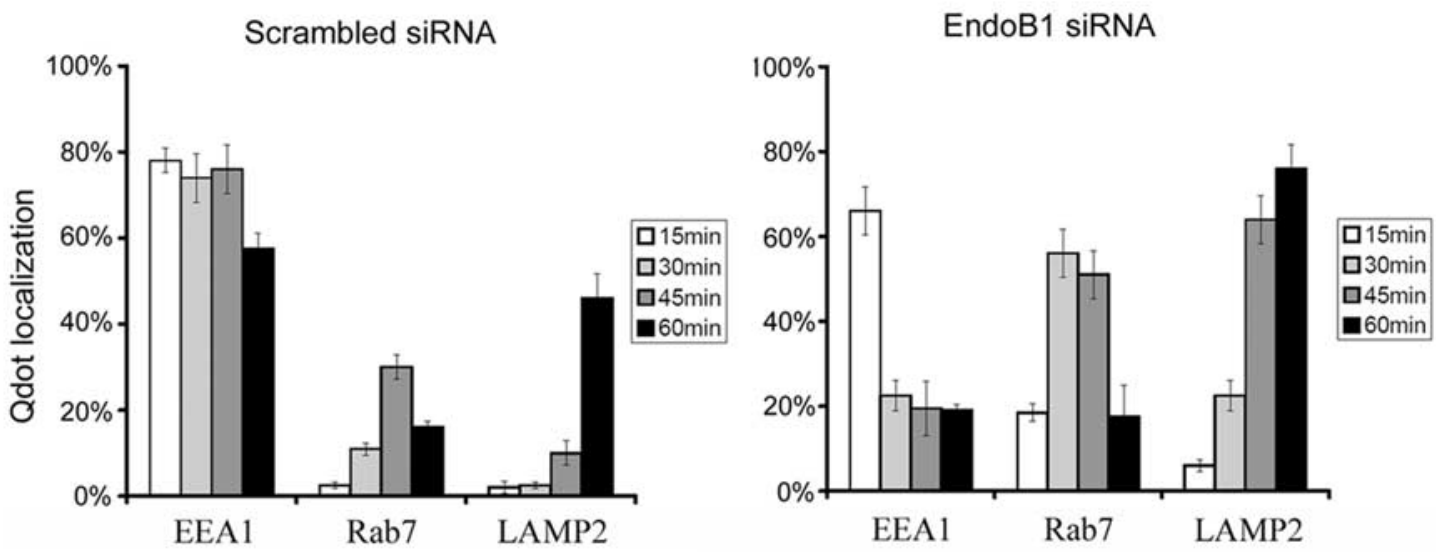

B.

Qdot-NGF 45 min

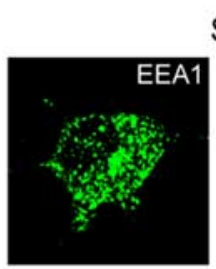

Scrambled siRNA
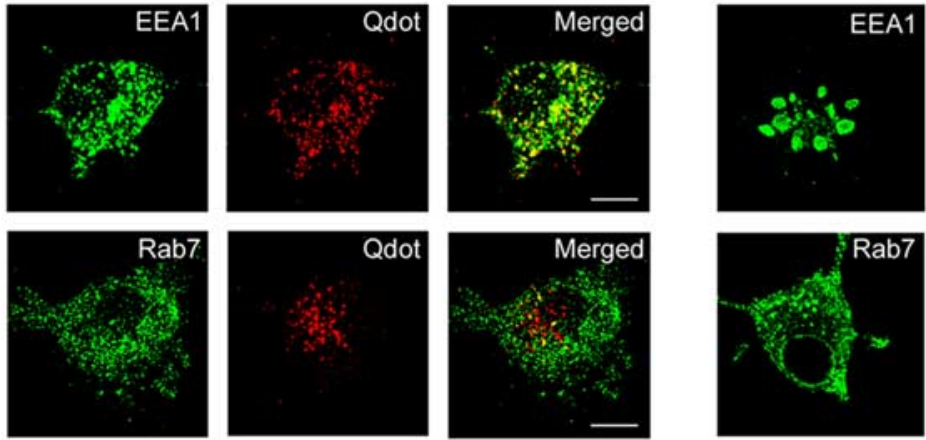

EndoB1 siRnA
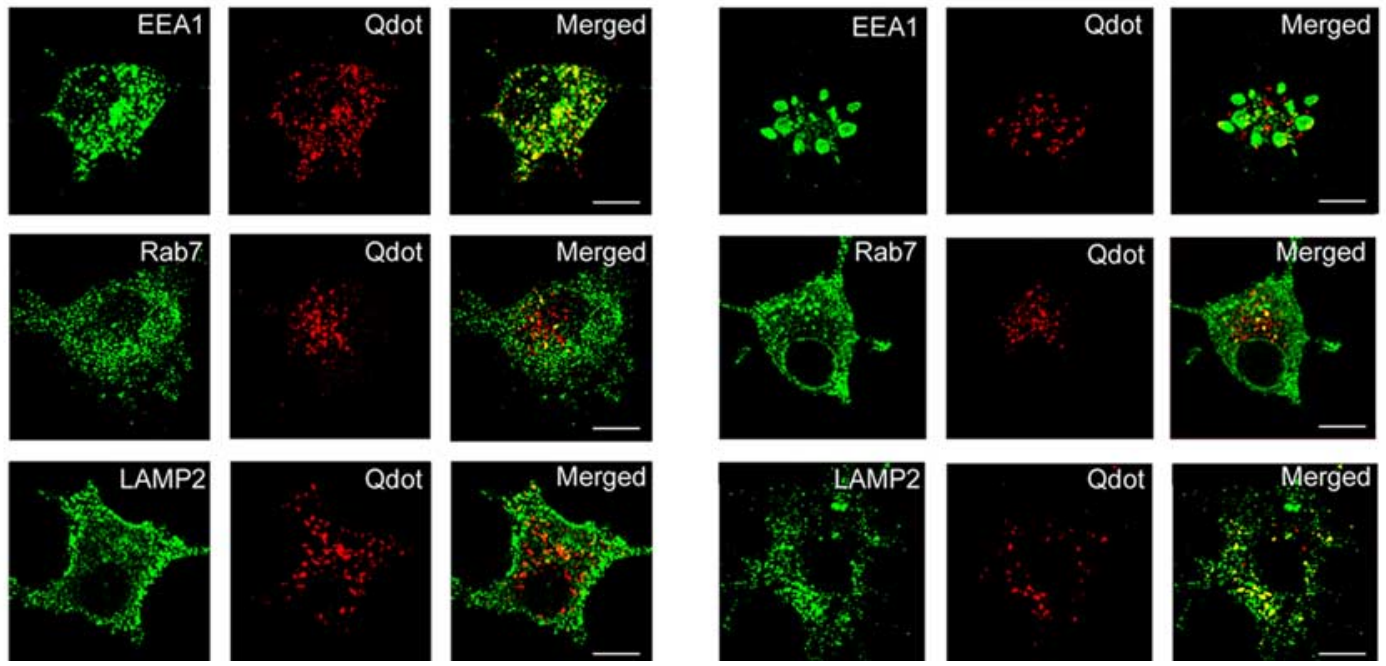

Figure 3. Knockdown of endophilin B1 favored trafficking of NGF to late endosomes and lysosomes. $A$, Left, Distribution of Qdot-NGF in scramble siRNA-transfected PC12 cells. To observe the trafficking of internalized NGF, PC12 cells were treated with Qdot-NGF and fixed at various time points after treatment. Cells were then stained against EEA1 to visualize early endosomes, Rab7 to visualize late endosomes, and LAMP2 to visualize lysosomes. We found that Qdot-NGF was initially sorted to early endosomes. Qdot-NGF was then present in late endosomes. By 45-60 min, a marked increase in the proportion of Qdot-NGF colocalized with lysosomes. Right, Distribution of Qdot-NGF in endophilin B1 siRNA-transfected PC12 cells. Knockdown of endophilin B1 expression markedly reduced the proportion of Qdot-NGF colocalizing with EEA1. The proportion colocalizing with Rab7 and LAMP2 was markedly enhanced. Our observations indicate that knockdown of endophilin B1 favored sorting of Qdot-NGF to late endosomes and lysosomes. Error bars indicate SD. B, Photomicrographs depicting the colocalization of Qdot-NGF with early endosomes, late endosomes, and lysosomes 45 min after treatment with Qdot-NGF in scrambled siRNA- (left) and endophilin B1-siRNA-transfected PC12 cells (right). Scale bars, $10 \mu \mathrm{m}$.

with the significant reduction in endophilin B1 protein levels after transfection with endophilin B1 siRNA (Fig. $2 B$ ), endophilin B1 transcripts were also markedly reduced (Fig. 4D). In addition, we found that NGF treatment enhanced transcription of transcription factor Egr-1, in accordance with a previous report (Harada et al., 2001) (Fig. 4D). Remarkably, reduction of endophilin B1 levels attenuated NGF-induced increase in Egr-1 transcript after $1 \mathrm{~d}$ of NGF treatment (Fig. 4D). Our observations reveal that endophilin B1 contributes to regulation of NGFinduced gene transcription in PC12 cells.

Reduction of endophilin B1 levels inhibited NGF-induced neurite outgrowth in PC12 cells and superior cervical ganglion neurons

Because knockdown of endophilin B1 markedly reduced TrkA level, TrkA signaling on endosomes and NGF-induced gene transcription, we investigated whether knockdown of en- dophilin B1 affects downstream functions of TrkA. It has long been established that NGF stimulation of PC12 cells results in neurite outgrowth. We thus examined whether knockdown of endophilin B1 affected NGF-induced neurite outgrowth in PC12 cells. Remarkably, we found that knockdown of endophilin B1 essentially abolished neurite outgrowth from PC12 cells after NGF stimulation (Fig. 5A). Furthermore, when knockdown of endophilin B1, achieved by the transfection of pSUPER-endophilin B1 shRNA, was reversed through cotransfecting an endophilin B1 mutant that evades degradation by endophilin B1 shRNA (shRNA-resistant endophilin B1), the inhibition of NGF-induced neurite outgrowth by endophilin B1 knockdown was markedly attenuated (Fig. 5B). These findings strongly suggest that endophilin $\mathrm{B} 1$ is required for NGF-induced neurite outgrowth in PC12 cells.

To further establish the involvement of endophilin B1 in NGF trafficking and NGF-induced neurite outgrowth, we ex- 
A. Scrambled siRNA EndoB1 siRNA

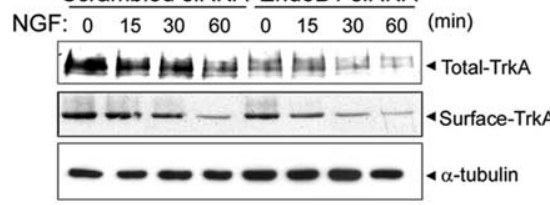

B.

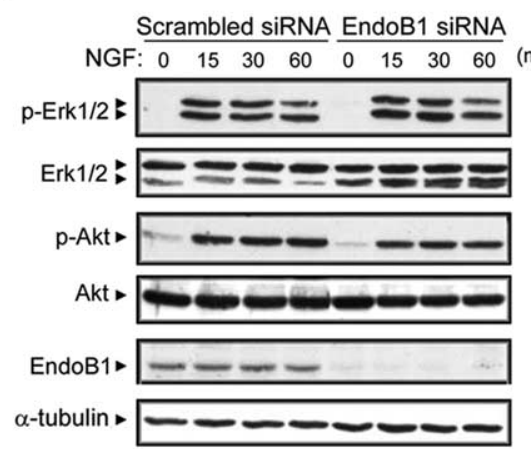

D.

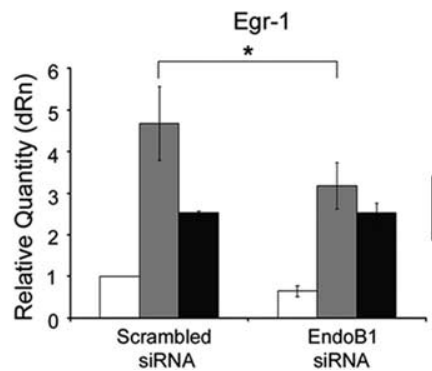

Egr-1
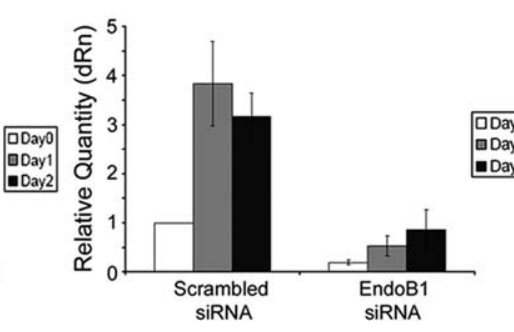

Figure 4. Knockdown of endophilin B1 triggered accelerated degradation of TrkA and reduced TrkA signaling on endosomes and NGF-induced gene transcription. $A$, Knockdown of endophilin B1 expression resulted in reduced basal level of total and surface TrkA and accelerated NGF-induced TrkA downregulation. PC12 cells transfected with scrambled or endophilin B1 siRNA were treated with NGF for different periods, and the amount of total and surface TrkA was examined. Knockdown of endophilin B1 reduced total and surface TrkA levels. The histogram depicts changes in TrkA levels in scrambled or endophilin B1 knockdown cells after NGF treatment. Values were normalized to the respective TrkA levels before NGF treatment. $\boldsymbol{B}$, Knockdown of endophilin B1 had negligible effect on the activation of Erk1/2 and Akt in the whole-cell lysates of PC12 cells. $\alpha$-Tubulin serves as a loading control. C, Knockdown of endophilin B1 markedly reduced activation of TrkA and Erk1/2 in endosomal fractions. The endosomal fraction (fraction 4) was extracted after centrifugation with a sucrose gradient. Knockdown of endophilin B1 resulted in a marked decrease in total TrkA and Erk1/2 level in the endosomal fraction, together with a decrease in phospho-TrkA and phospho-Erk1/2 level. Akt signaling, however, was not affected. Rap1 serves as a marker for early endosomes. D, Knockdown of endophilin B1 attenuated NGF-induced gene transcription in PC12 cells. NGF treatment has been demonstrated to induce expression of transcription factor Egr-1. Transfection with endophilin B1 siRNA nearly abolished endophilin B1 transcripts level in PC12 cells. Interestingly, knockdown of endophilin B1 expression markedly attenuated NGF-induced Egr-1 expression after $1 \mathrm{~d}$ of NGF treatment. Error bars indicate SD. ${ }^{*} p<0.05$.

amined the effect of knocking down endophilin B1 expression in primary cultures of SCG neurons, which are abundant in TrkA expression. Contrary to our previous observations in PC1 2 cells, Qdot-NGF is trafficked slightly differently in SCG neurons. For example, a much lower percentage of Qdot-NGF was observed in EEA1, Rab7, or LAMP2-positive vesicles compared with that observed in PC12 cells (Fig. 6A). In addition, Qdot-NGF was apparently trafficked at a much slower rate in SCG neurons. Although a significant portion of Qdot-NGF colocalized with LAMP2-positive lysosomes by $60 \mathrm{~min}$ of Qdot-NGF treatment in PC12 cells (Fig. 3A), <5\% of Qdot-
NGF colocalized with LAMP2-positive vesicles at $120 \mathrm{~min}$ of Qdot-NGF treatment in SCG neurons (Fig. 6A). Despite the difference observed between SCG neurons and PC12 cells, nonetheless, knockdown of endophilin B1 similarly induced a redistribution of Qdot-NGF to Rab7- or LAMP2-positive vesicles at 60 and 120 min of Qdot-NGF treatment (Fig. 6A). Furthermore, knockdown of endophilin B1 similarly inhibited neurite growth in SCG neurons, as reflected by the decreased neurite length and neurite number (Fig. 6B). These observations collectively reveal that similar to our observations in PC12 cells, knockdown of endophilin B1 favors redistribution of Qdot-NGF/TrkA to late endosomes and lysosomes, in addition to inhibiting neurite extension in SCG neurons.

\section{Discussion}

In the current study, we have discovered an unanticipated function of endophilin B1 in neurons by identifying endophilin B1 as a novel regulator of NGF/TrkA trafficking and function. Using immunoprecipitation studies and immunocytochemical analysis, we revealed the localization of endophilin B1 to early endosomes in neurons, and demonstrated its association with early endosome marker EEA1. Remarkably, knockdown of endophilin B1 induces precocious targeting of NGF, likely in a complex with TrkA, to late endosomes and lysosomes, thereby enhancing degradation of TrkA. In addition, endophilin B1 knockdown markedly reduces TrkA level, and inhibits Erk1/2 recruitment and activation on endosomes. This is accompanied by attenuation of NGFinduced gene transcription and neurite outgrowth. Our findings have therefore revealed a pivotal role of endophilin $\mathrm{B} 1$ in NGF/TrkA trafficking and downstream functions through controlling TrkA level and initiation of downstream signaling on endosomes.

What could be the mechanisms by which endophilin B1 affects trafficking of NGF/TrkA? Given the colocalization and interaction of endophilin B1 with early endosome marker EEA1, it is tempting to speculate that it may act as a general regulator of the endocytic pathways, thereby affecting intracellular trafficking of a multitude of surface receptors. Nonetheless, by examining the trafficking of transferrin/transferrin receptor and NGF/TrkA, we unexpectedly revealed that, whereas knockdown of endophilin B1 affected trafficking of TrkA, trafficking of transferrin was negligibly affected. Indeed, whereas endophilin B1 interacts with TrkA both before and after NGF treatment, no association between endophilin 
B1 and transferrin receptor was detected. It is thus plausible that part of the selectivity was conferred by differential binding of endophilin B1 with different surface receptors. Several observations provided other hints as to how endophilin B1 may affect NGF/TrkA trafficking. First, association of endophilin B1 with both EEA1 and TrkA was augmented after NGF treatment. It is possible that, on NGF treatment, the enhanced association of endophilin B1 with TrkA and EEA1 enables endophilin B1 to act as a carrier to bring TrkA and EEA1 into the same compartment. Indeed, whereas NGF treatment induces colocalization of EEA1 with TrkA and Qdot-NGF in control cells, colocalization of TrkA and Qdot-NGF with EEA1 was markedly reduced in the absence of endophilin B1 (Fig. 2C). Second, we unexpectedly found that knockdown of endophilin B1 triggers enlargement of EEA1-positive early endosomes, only after NGF stimulation. This observation suggests that endophilin B1 does not affect the morphology of early endosomes under basal condition, but only specifically modulates the size of early endosomes created in response to NGF stimulation. It thus appears that, after stimulation with NGF, TrkA, endophilin B1, and EEA1 are recruited to the same compartment, presumably early endosomes, where the presence of endophilin B1 is critical for controlling the size of early endosomes.

Does the enlargement of early endosomes triggered by endophilin B1 knockdown directly contribute to the precocious targeting of NGF/TrkA to late endosomes and lysosomes? Several previous studies have documented the presence of enlarged early endosomes (Ceresa et al., 2001; Li et al., 2002; Simpson et al., 2004), but its significance on protein trafficking in the endocytic pathway remains mostly unknown. The presence of enlarged endosomes, likely containing NGF, could possibly be attributable to enhanced endocytosis of NGF/TrkA or defective fission and movement of vesicles with NGF/TrkA to other compartments such as late endosomes or recycling endosomes. Nonetheless, because enlarged early endosomes are accompanied by precocious targeting to late endosomes, it is unlikely that the enlarged early endosomes was attributable to defective movement of cargo-containing vesicles to late endosomes. It is thus possible that the enlarged early endosomes may not directly contribute to the accelerated lysosomal targeting in endophilin B1 knockdown cells. Rather, the enlarged endosomes observed after knockdown of endophilin B1 (Fig. 4A) may represent enhanced internalization or defective recycling of NGF/ TrkA to the surface, because it was recently reported that internalized TrkA and TrkB recycle at an average half-life of $7 \mathrm{~min}$ (Chen et al., 2005). In support of this possibility, we found that
A.
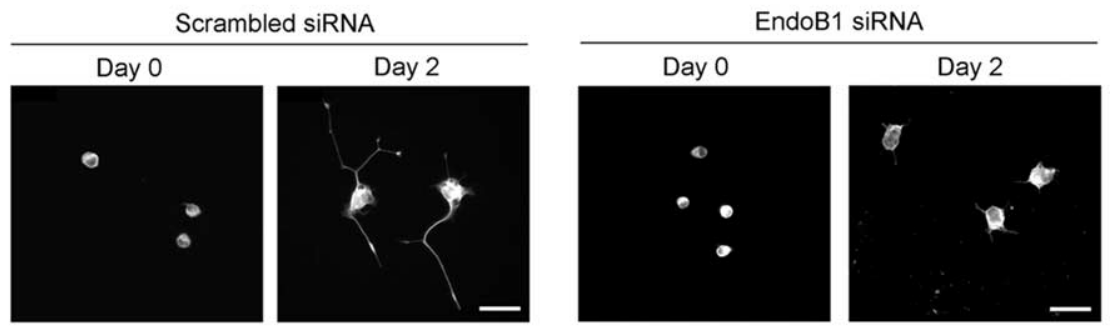

Longest neurite length

Average neurite number
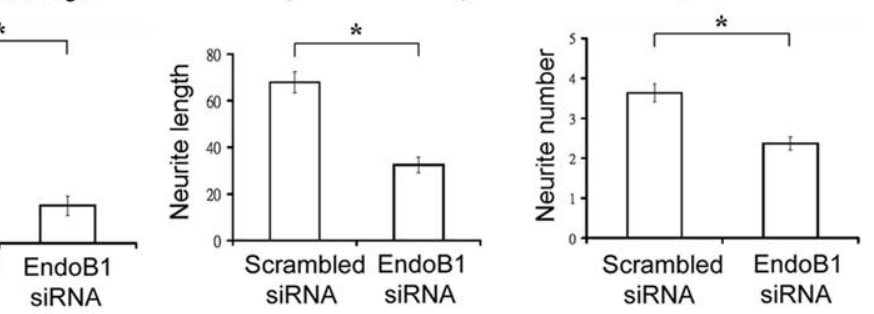

pSUPER-EndoB1 + GFP pSUPER-EndoB1 + EndoB1
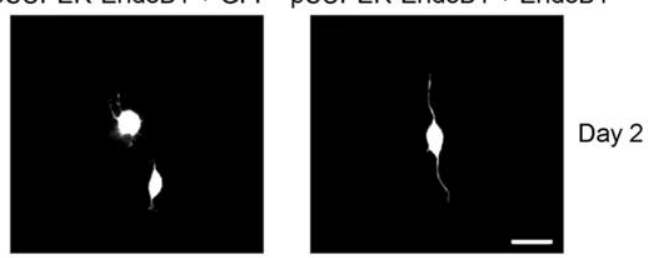

Longest neurite length

Average neurite number
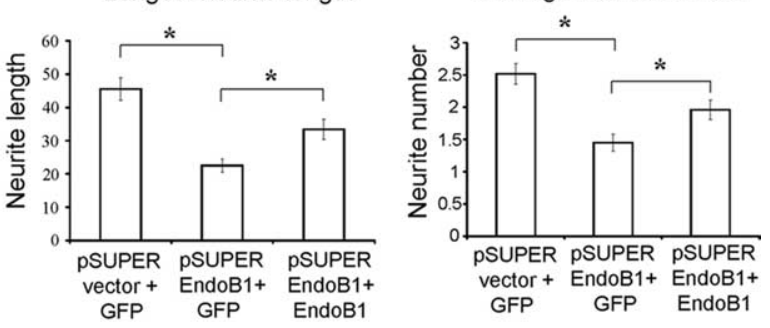

Figure 5. Knockdown of endophilin B1 inhibited NGF-induced neurite outgrowth in PC12 cells.A, Knockdown of endophilin B1 inhibited NGF-induced neurite outgrowth in PC12 cells. PC12 cells transfected with scrambled or endophilin B1-siRNA were

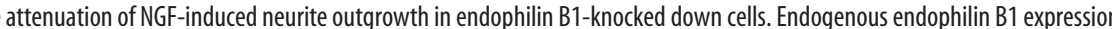
insensitive to endophilin B1 shRNA (EndoB1) partially reversed the inhibition of NGF-induced neurite outgrowth in PC12 cells. Error bars indicate SD. ${ }^{*} p<0.05$. Scale bars, $20 \mu \mathrm{m}$.

knockdown of endophilin B1 reduced colocalization of QdotNGF/TrkA with Rab4 and Rab11, markers of recycling endosomes (supplemental Fig. 3, available at www.jneurosci.org as supplemental material). This observation suggests that the enlarged endosomes may be partially attributed to attenuated NGF/ TrkA recycling in endophilin B1-knocked down cells. Additional studies will be required to consolidate the effect of endophilin B1 on NGF/TrkA recycling.

In conclusion, we identified an unexpected role of endophilin B1 in NGF trafficking and functions. Through regulation of NGF/TrkA endocytic trafficking, endophilin B1 regulates TrkA level and signaling cascade on endosomes to mediate downstream TrkA functions. Given the potential role of endophilin B1 in the regulation of mitochondrial morphology and apoptosis in fibroblast, it would be interesting to explore whether endophilin $\mathrm{B} 1$ is also implicated in the regulation of neuronal survival and 
A.
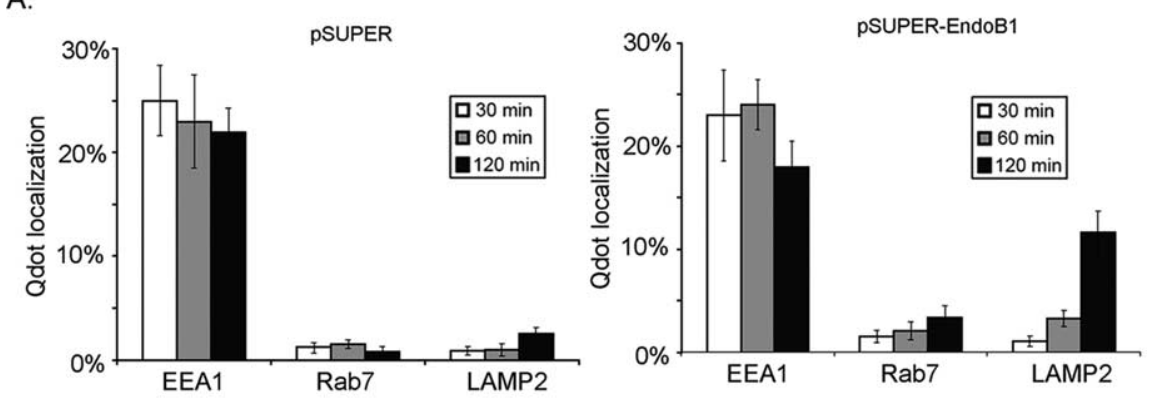

B.

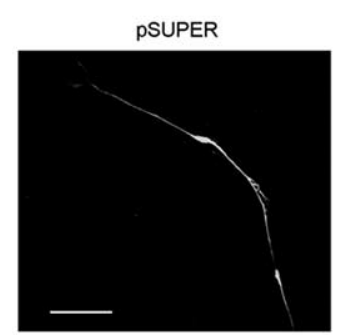

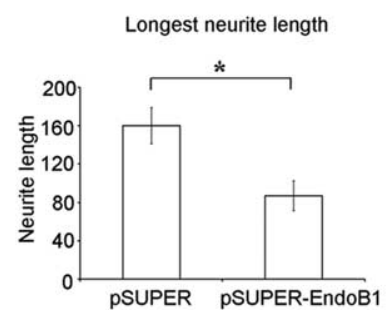

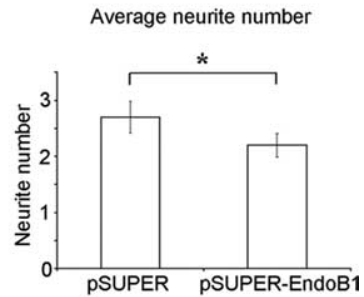

Cui B, Wu C, Chen L, Ramirez A, Bearer EL, Li WP, Mobley WC, Chu S (2007) One at a time, live tracking of NGF axonal transport using quantum dots. Proc Natl Acad Sci U S A 104:13666-13671.

Delcroix JD, Valletta JS, Wu C, Hunt SJ, Kowal AS, Mobley WC (2003) NGF signaling in sensory neurons: evidence that early endosomes carry NGF retrograde signals. Neuron 39:69-84.

Farsad K, Ringstad N, Takei K, Floyd SR, Rose K, De Camilli P (2001) Generation of high curvature membranes mediated by direct endophilin bilayer interactions. J Cell Biol 155:193-200.

Gáborik Z, Hunyady L (2004) Intracellular trafficking of hormone receptors. Trends Endocrinol Metab 15:286-293.

Ginty DD, Segal RA (2002) Retrograde neurotrophin signaling: Trk-ing along the axon. Curr Opin Neurobiol 12:268-274.

Harada T, Morooka T, Ogawa S, Nishida E (2001) ERK induces p35, a neuron-specific activator of Cdk5, through induction of Egr1. Nat Cell Biol 3:453-459.

Heerssen HM, Pazyra MF, Segal RA (2004) Dynein motors transport activated Trks to promote survival of target-dependent neurons. Nat Neurosci 7:596-604.

Howe CL, Mobley WC (2005) Long-distance retrograde neurotrophic signaling. Curr Opin Neurobiol 15:40-48.

Huang EJ, Reichardt LF (2003) Trk receptors: roles in neuronal signal transduction. Annu Rev Biochem 72:609-642.

Huttner WB, Schiebler W, Greengard P, De Camilli P (1983) Synapsin I (protein I), a nerve terminal-specific phosphoprotein. III. Its association with synaptic vesicles studied in a highly purified synaptic vesicle preparation. J Cell Biol 96:1374-1388.

Karbowski M, Jeong SY, Youle RJ (2004) Endophilin $\mathrm{B} 1$ is required for the maintenance of mitochondrial morphology. J Cell Biol 166:1027-1039.

Kuruvilla R, Zweifel LS, Glebova NO, Lonze BE, Valdez G, Ye H, Ginty DD (2004) A neurotrophin signaling cascade coordinates sympa-

mitochondrial morphology in neurons. A recent study revealed that endophilin B1 is also implicated in the regulation of autophagy in fibroblast (Takahashi et al., 2007). Whether endophilin B1 may regulate the survival promoting effect of neurotrophins by controlling the trafficking of Trk receptors, or whether it may concurrently temper with the balance of survival and death signals by affecting mitochondrial functions or autophagy would be an interesting area for additional investigation.

\section{References}

Bronfman FC, Tcherpakov M, Jovin TM, Fainzilber M (2003) Ligandinduced internalization of the $\mathrm{p} 75$ neurotrophin receptor: a slow route to the signaling endosome. J Neurosci 23:3209-3220.

Ceresa BP, Lotscher M, Schmid SL (2001) Receptor and membrane recycling can occur with unaltered efficiency despite dramatic Rab5(q79l)-induced changes in endosome geometry. J Biol Chem 276:9649-9654.

Chen ZY, Ieraci A, Tanowitz M, Lee FS (2005) A novel endocytic recycling signal distinguishes biological responses of Trk neurotrophin receptors. Mol Biol Cell 16:5761-5772.

Cuddeback SM, Yamaguchi H, Komatsu K, Miyashita T, Yamada M, Wu C, Singh S, Wang HG (2001) Molecular cloning and characterization of Bif-1. A novel Src homology 3 domain-containing protein that associates with Bax. J Biol Chem 276:20559-20565. thetic neuron development through differential control of TrkA trafficking and retrograde signaling. Cell 118:243-255.

Li Y, Chin LS, Levey AI, Li L (2002) Huntingtin-associated protein 1 interacts with hepatocyte growth factor-regulated tyrosine kinase substrate and functions in endosomal trafficking. J Biol Chem 277:28212-28221.

Liu Y, Cheng K, Gong K, Fu AK, Ip NY (2006) Pctaire1 phosphorylates $\mathrm{N}$-ethylmaleimide sensitive fusion protein: implications in the regulation of its hexamerization and exocytosis. J Biol Chem 281:9852-9858.

Miaczynska M, Zerial M (2002) Mosaic organization of the endocytic pathway. Exp Cell Res 272:8-14.

Modregger J, Schmidt AA, Ritter B, Huttner WB, Plomann M (2003) Characterization of Endophilin B1b, a brain-specific membrane-associated lysophosphatidic acid acyl transferase with properties distinct from endophilin A1. J Biol Chem 278:4160-4167.

Neet KE, Campenot RB (2001) Receptor binding, internalization, and retrograde transport of neurotrophic factors. Cell Mol Life Sci 58:1021-1035.

Ng YP, He W, Ip NY (2003) Leukemia inhibitory factor receptor signaling negatively modulates nerve growth factor-induced neurite outgrowth in PC12 cells and sympathetic neurons. J Biol Chem 278:38731-38739.

Niethammer M, Smith DS, Ayala R, Peng J, Ko J, Lee MS, Morabito M, Tsai 
LH (2000) NUDEL is a novel Cdk5 substrate that associates with LIS1 and cytoplasmic dynein. Neuron 28:697-711.

Rong J, McGuire JR, Fang ZH, Sheng G, Shin JY, Li SH, Li XJ (2006) Regulation of intracellular trafficking of huntingtin-associated protein-1 is critical for TrkA protein levels and neurite outgrowth. J Neurosci 26:6019-6030

Simpson JC, Griffiths G, Wessling-Resnick M, Fransen JA, Bennett H, Jones AT (2004) A role for the small GTPase Rab21 in the early endocytic pathway. J Cell Sci 117:6297-6311.

Takahashi Y, Karbowski M, Yamaguchi H, Kazi A, Wu J, Sebti SM, Youle RJ, Wang HG (2005) Loss of Bif-1 suppresses Bax/Bak conformational change and mitochondrial apoptosis. Mol Cell Biol 25:9369-9382.
Takahashi Y, Coppola D, Matsushita N, Cualing HD, Sun M, Sato Y, Liang C, Jung JU, Cheng JQ, Mul JJ, Pledger WJ, Wang HG (2007) Bif-1 interacts with Beclin 1 through UVRAG and regulates autophagy and tumorigenesis. Nat Cell Biol 9:1142-1151.

Volpicelli LA, Lah JJ, Fang G, Goldenring JR, Levey AI (2002) Rab1la and myosin $\mathrm{Vb}$ regulate recycling of the M4 muscarinic acetylcholine receptor. J Neurosci 22:9776-9784.

Wu C, Lai CF, Mobley WC (2001) Nerve growth factor activates persistent Rap1 signaling in endosomes. J Neurosci 21:5406-5416.

Ye H, Kuruvilla R, Zweifel LS, Ginty DD (2003) Evidence in support of signaling endosome-based retrograde survival of sympathetic neurons. Neuron 39:57-68. 